within four hours) who completed alcohol treatment. A comparison group $(\mathrm{n}=806)$ of those with one or two violations who did not attend alcohol treatment was created by matching to the treatment group on demographic and risk factors.

Results The program keys most correlated with higher interlock rates were having a requirement to instal interlocks $(r=0.63)$, and monitoring to ensure interlocks are installed and used $(\mathrm{r}=0.56)$. Incorporating alcohol treatment into an interlock program was effective with the treatment group experiencing $32 \%$ lower recidivism following interlock removal compared with the non-treatment group.

Conclusions Strengthening program keys and incorporating treatment into interlock programs increases use of interlocks and reduces AID re-arrest.

\section{MARIJUANA USE AND EXCESS RISK OF INJURY EVENTS: FINDINGS FROM A LARGE PROSPECTIVE COHORT STUDY}

${ }^{1}$ Shanthi Ameratunga, ${ }^{1}$ Roshini Peiris-John, ${ }^{1}$ Papaarangi Reid, ${ }^{1}$ Arier Lee, ${ }^{2}$ Gordon S Smith, ${ }^{3}$ Robyn Norton. ${ }^{1}$ University of Auckland, New Zealand; ${ }^{2}$ University of Maryland, USA; ${ }^{3}$ The George Institute for Global Health, Australia

\subsection{6/injuryprev-2016-042156.301}

Background Marijuana is one of most widely used illicit substances globally. The risks of injury experienced by marijuana users relative to non-users have been poorly quantified in prospective studies. We investigated the associations between marijuana use and fatal or hospitalised injury in the New Zealand Blood Donors' Health Study.

Methods At recruitment to this prospective cohort study in 1998-1999, the 22,389 participants completed a self-report survey on demographic, personal and lifestyle questions including the frequency of marijuana use. Using unique identifiers, these data were record-linked to national mortality and hospital discharge databases to ascertain participants' injury-related admissions or deaths from recruitment to 31 December 2014. The associations between marijuana use and injury were investigated using Cox proportional hazards analyses, adjusting for sociodemographic and confounding factors.

Results At baseline, $15 \%$ of participants of the cohort reported marijuana use in the preceding 12 months. During a median follow-up period of 16.8 years, 3,693 incident injury cases $(3,651$ non-fatal and 63 fatal) occurred, the majority of which were falls (1204 incident cases) and motor vehicle crashes (779 incident cases). Compared to non-users, marijuana users were more likely to experience injuries related to self-harm (adjusted HR 2.00, 95\% CI: 1.39-2.89), assault (adjusted HR 1.83, 95\% CI: $1.27-$ 2.66), motor vehicle crashes (adjusted HR 1.35, 95\% CI: 1.091.69), or unintended cutting or piercing trauma (adjusted HR 1.76, 95\% CI: 1.31-2.35). Risk estimates were greater with more frequent use of marijuana.

Conclusions Relative to non-users, participants who used marijuana were at increased risk of most major types of injuries, with an apparent dose-response effect. The pathways and correlates of harm underlying the increased risks of injury (particularly selfharm and assault) associated with marijuana use, require public health attention.

\section{ALCOHOL OUTLET DENSITY AND HOSPITAL ADMISSIONS FOR ALCOHOL-RELATED INJURY: AN ELECTRONIC RECORD-LINKED COHORT STUDY}

${ }^{1}$ Sarah Rodgers, ${ }^{2}$ Daniel Farewell, ${ }^{2}$ Frank Dunstan, ${ }^{2}$ James White, ${ }^{3}$ Scott Orford, ${ }^{2}$ Jennifer Morgan, ${ }^{1}$ Richard Fry, ${ }^{1}$ Ronan Lyons, ${ }^{2}$ David Fone. ${ }^{1}$ Farr Institute, Swansea University, UK; ${ }^{2}$ Institute of Primary Care and Public Health, Cardiff University, UK; ${ }^{3}$ School of Planning and Geography, Cardiff University, UK

\subsection{6/injuryprev-2016-042156.302}

Background Little is known on the longitudinal relationship between alcohol availability and the risk of admission to hospital for an alcohol-related injury. We aimed to quantify this relationship using observational record-linked data in a total adult population cohort in Wales, UK; population 2.5 million aged 16 years and over.

Methods Data sources included licensed outlets held by the 22 local authorities in Wales under The Licensing Act 2003 for each quarter between 2006 and 2011. Alcohol outlet density was estimated for Census geography small areas $(n=1896$ divided into quintiles of equal counts) based on the mean network walking distances between each household and alcohol outlet within a 10 minute walk. Hospital admissions 2006-2011 from the Patient Episode Database for Wales (PEDW) for wholly alcohol-related conditions were anonymously record-linked to the Welsh Demographic Service age-sex register within the Secure Anonymised Information Linkage Databank. Injury admissions were defined by ICD-10 codes S00-99 and T00-19 linked to an alcohol code. Longitudinal statistical analysis used Cox regression models of hospital admissions as a function of outlet density at baseline, adjusting for confounding variables of age, sex and small area deprivation and settlement type, and censoring for death, migration and other cause admission.

Results Alcohol outlet density at baseline was significantly associated with emergency hospital admission; the hazard ratio (HR) of an alcohol-related admission $(n=25,722)$ for living in the highest compared with the lowest quintile of outlet density was 1.17 (95\% CI: 1.11, 1.23). The risk was higher for alcohol-related admissions with injury ( $\mathrm{n}=4,308$; HR 1.27, 95\% CI: 1.13 , 1.42).

Conclusions Higher alcohol outlet availability was associated with more emergency hospital admissions for alcohol-related injury. This suggests that restricting the density of alcohol outlets within walking distance from home may improve population health.

\section{DOES INCREASING ALCOHOL TAXES ALWAYS REDUCE ALCOHOL-RELATED CRASHES: MARYLAND SALES TAX INCREASE?}

${ }^{1}$ Marie-Claude Lavoie, ${ }^{1}$ Gordon S Smith, ${ }^{1}$ Patricia Langenberg, ${ }^{2}$ Andres Villaveces, ${ }^{1}$ Patricia Dischinger, ${ }^{1}$ Linda Simoni-Wastila, ${ }^{1}$ Kathleen Hoke. ${ }^{1}$ University of Maryland, USA; ${ }^{2}$ World Bank, Washington, DC, USA

\subsection{6/injuryprev-2016-042156.303}

Background The sales tax on all alcoholic drink was raised in Maryland from 6 to 9\% on July 1, 2011. This study evaluates the effects of the alcohol sales tax increase on the rate of alcoholinvolved drivers involved in fatal crashes. 
Methods Study population: All drivers involved in a fatal motor vehicle crash in MD January 1, 2001 to December 31, 2013: 126 monthly data points from police reports pre-intervention and 30 post- intervention. Using an interrupted time series analysis we examined the effect of the tax on all alcohol-involved drivers in fatal crashes and fatally injured alcohol-involved drivers. Rates were calculated per 100,000 population, 100,000 licensed drivers, and 100 million vehicle miles travelled.

Results 6,967 crashes involving 10,777 drivers resulted in 4,642 driver fatalities. Unadjusted analysis found a significant $15 \%$ reduction in rate of alcohol-impaired drivers with BAC $>0.08 \mathrm{~g} /$ $\mathrm{dL}$ per vehicle miles travelled after the tax increase. Significant reduction was also observed for alcohol-positive drivers and for fatally injured BAC positive drivers. After adjusting for linear trend, seasonality, and unemployment, the immediate effect of the 2011 alcohol sales tax increase in was not significant. However, we did observe a significantly larger decline in the rates of alcohol-impaired drivers and alcohol-related fatalities with BAC $>0.08 \mathrm{~g} / \mathrm{dL}$ as indicated by the significant gradual decline in the slope after the introduction of the 2011 alcohol sales tax increase.

Conclusions: While there was no immediate reduction in the rate of alcohol-impaired drivers following a $3 \%$ increase in alcohol sales tax, there was a significantly larger decline in the rates of alcohol-involved fatalities at BAC $>0.08 \mathrm{~g} / \mathrm{dL}$ following the intervention, suggesting a gradual delayed effect. Other studies find an effect with excise taxes but this is the first study to evaluate the impact of sales taxes.

\section{Trauma Care and Rehabilitation}

\section{Parallel 2.5}

\section{4 "THAT'S WHAT YOU DO FOR PEOPLE YOU LOVE": A QUALITATIVE STUDY OF SOCIAL SUPPORT AND RECOVERY FROM MUSCULOSKELETAL INJURY}

Khic-Houy Prang, Sharon Newnam, Janneke Berecki-Gisolf. Monash Injury Research Institute, Monash University, Australia

\subsection{6/injuryprev-2016-042156.304}

Background Social support has been identified as a key factor in facilitating better health outcomes following injury. However, research has primarily focused on the role of social support from the perspective of the person experiencing an injury. Limited research has examined the experiences of the family members/ friends to a person with injury. This study aims to explore the perceptions and experiences of social support and recovery following a transport-related musculoskeletal injury (MSI) in a population of injured persons and their family members/friends.

Methods This study was conducted using a phenomenology qualitative research design. In-depth semi-structured interviews were conducted with ten persons with MSI, recruited via the Transport Accident Commission (TAC) in Victoria, Australia. Seven family members/friends were also interviewed. Interviews were audiorecorded and transcribed verbatim. The data was analysed using thematic analysis.

Results Several themes were identified including: (i) key sources of support, (ii) types of support received, (iii) relationship development and (iv) challenges of providing and receiving support. The presence of social support was perceived as fundamental to recovery. Different members of the social network provided different types of support. Iterative changes in relationships and barriers in providing and receiving support following the injury were noted.

Conclusions This study provides valuable insights into the perceptions and experiences of social support and recovery from MSI. The study revealed complexities in the interactions between persons with MSI and their family members/friends, in particularly related to caregiving demands. The findings of this study have implication for involving and supporting family members/ friends in the planning and implementation of treatment plan.

\section{TRAUMA CARE SYSTEM MATURITY IN INDIA: EVALUATION USING WHO MATURITY INDEX AND GEOSPATIAL ANALYSES}

${ }^{1} \mathrm{BM}$ Asheel, ${ }^{2} \mathrm{~K}$ Beebi, ${ }^{2} \mathrm{~K}$ Jayeshlal, M Nambiar. ${ }^{1}$ National Health Mission, Kerala, India; ${ }^{2}$ Directorate of Health Services, Kerala, India

\subsection{6/injuryprev-2016-042156.305}

Background In the context of recent emphasis on developing trauma care systems globally, a Pan-India trauma care system is getting ready. Given the well known regional disparities in health systems in India, evaluation of its parts (states) becomes important if the sum of the parts (the Nation) needs to develop a system in an equitable way. Trauma System Maturity Index (TSMI) and other guidelines by WHO, along with Geographical Information System (GIS) provides an opportunity for such evaluation and evidence based policy making.

Methods A cross sectional analytical study was done to grade trauma care system maturity of 10 representative states in India. Four major components of trauma system were assessed, including pre-hospital care, education and training, facility based trauma care and quality assurance; using a structured schedule prepared in line with WHO TSMI and 7 major guidelines for trauma care by WHO. In addition to this, GIS based analyses were done to assess the geographical distribution of trauma care facilities. The GIS data was analysed using ArcGIS version 9.1 and data for TSMI analysed using SPSS version 17.0.

Results While most of the state trauma care systems were graded level 2 for prehospital care and quality assurance, almost all systems graded 1 for facility based trauma care. Host of variables within the components were analysed and critical areas were identified where in small initiatives would evolve the trauma system to next level of maturity grading. GIS based analyses showed that even with in the states the access to trauma care services is alarmingly inequitable. Better grade in TSMI translated as better outcome in terms of trauma related mortality.

Conclusions The trauma care systems in different states are in diverse phases of evolution and their TSMI grade reflects their system performance. Study also projects successful examples which can be adapted by other states and countries with similar policy environment.

\section{PRE-HOSPITAL CARE IN OMAN: RESULTS FROM HOSPITAL BASED TRAUMA REGISTRY}

${ }^{1}$ Amber Mehmood, ${ }^{1}$ Katharine A Allen, ${ }^{1} J$ Joseph Salami, ${ }^{2}$ Ammar Al Kashmiri, ${ }^{2}$ Ali Al Busaidi, 'Kent Stevens, 'Adnan A Hyder. 'Johns Hopkins Bloomberg School of Public Health, USA; ${ }^{2}$ Ministry of Health, Sultanate of Oman

10.1136/injuryprev-2016-042156.306 